\title{
Multi-targeted priming for genome-wide gene expression assays
}

\author{
Aleksandra B Adomas ${ }^{1}$, Francesc Lopez-Giraldez ${ }^{1}$, Travis A Clark¹, Zheng Wang ${ }^{1}$, Jeffrey P Townsend ${ }^{2 *}$
}

\begin{abstract}
Background: Complementary approaches to assaying global gene expression are needed to assess gene expression in regions that are poorly assayed by current methodologies. A key component of nearly all gene expression assays is the reverse transcription of transcribed sequences that has traditionally been performed by priming the poly-A tails on many of the transcribed genes in eukaryotes with oligo-dT, or by priming RNA indiscriminately with random hexamers. We designed an algorithm to find common sequence motifs that were present within most protein-coding genes of Saccharomyces cerevisiae and of Neurospora crassa, but that were not present within their ribosomal RNA or transfer RNA genes. We then experimentally tested whether degenerately priming these motifs with multi-targeted primers improved the accuracy and completeness of transcriptomic assays.

Results: We discovered two multi-targeted primers that would prime a preponderance of genes in the genomes of Saccharomyces cerevisiae and Neurospora crassa while avoiding priming ribosomal RNA or transfer RNA. Examining the response of Saccharomyces cerevisiae to nitrogen deficiency and profiling Neurospora crassa early sexual development, we demonstrated that using multi-targeted primers in reverse transcription led to superior performance of microarray profiling and next-generation RNA tag sequencing. Priming with multi-targeted primers in addition to oligo-dT resulted in higher sensitivity, a larger number of well-measured genes and greater power to detect differences in gene expression.

Conclusions: Our results provide the most complete and detailed expression profiles of the yeast nitrogen starvation response and N. crassa early sexual development to date. Furthermore, our multi-targeting priming methodology for genome-wide gene expression assays provides selective targeting of multiple sequences and counter-selection against undesirable sequences, facilitating a more complete and precise assay of the transcribed sequences within the genome.
\end{abstract}

\section{Background}

Gene expression levels have been quantified by numerous procedures, including reverse transcription (RT)PCR [1], sequencing of expressed sequence tags [2], serial analysis of gene expression [3], microarray hybridization [4], and massively parallel signature sequencing [5]. Rapid development of platforms has improved throughput, but also generated strong demand for enhanced sensitivity and measurement accuracy. For nearly all expression assays, reverse transcription from

\footnotetext{
* Correspondence: Jeffrey.Townsend@Yale.edu

${ }^{2}$ Department of Ecology and Evolutionary Biology, Program in Computational Biology and Bioinformatics, and Microbiology Graduate Program, Yale University, 165 Prospect St, New Haven, CT 06511, USA Full list of author information is available at the end of the article
}

messenger RNA (mRNA) to complementary DNA (cDNA) is a key step of the process that contributes less experimental variance than biological growth and harvest, but greater experimental variance than hybridization [[6], but see also [7]]. Throughput of the reaction may be biased by secondary and tertiary structures of mRNA, affinities specific to the reverse transcriptase, inhibitors present in the sample, priming strategy, and variation in priming efficiency [8]. The most common priming strategies utilize oligo-dT primers, random primers, or gene-specific primers. When oligo-dT primers are used for reverse transcription, RNA secondary structure and variation in poly(A) tail length may result in gene amplification 3' bias [9]. Random primers, typically used in prokaryotic systems, fail to discriminate between 
mRNA and the preponderance of RNA in the form of ribosomal (rRNA) or transfer RNA (tRNA). Random hexamers, the most commonly employed, amplify only fraction of the transcriptome, comparing with random pentadecamers [10]. However, random oligonucleotides of any size also prime abundant rRNA and tRNA that can lead to high background and misleading signal.

Ribosomal RNA (rRNA) sequences in many prokaryotes are $\mathrm{GC}$ rich relative to the genome at large and are highly conserved. These properties have been used to design non-random hexamers (HD/DHTTTT) to prime reverse transcription reactions [11]. The result was a counter-selective synthesis of cDNA corresponding to mRNA from prokaryotic total RNA extractions. In contrast, application of gene-specific primers on a genomic scale requires synthesis of multiple primers. An algorithm to predict the minimal number of non-degenerate genome-directed primers that specifically anneal to all genes in a given genome has been designed and successfully applied in bacteria [12].

Another recently developed method relies on a collection of short, computationally selected oligonucleotides ('not-so-random' (NSR) primers) to obtain full-length, strand-specific representation of nonribosomal RNA transcripts [13]. Selective enrichment of non-rRNA targets was achieved by computationally subtracting rRNA priming sequences from a random hexamer library. The presence of rRNA and tRNA plagues most mRNA purification procedures due to their relative abundance, leading to non-specific interactions like rRNA adsorption to the oligo-dT matrix, or hybridization of rRNA and mRNA sequences [14].

Here we describe an alternate strategy, multi-targeted priming (MTP), that allows for selective amplification of chosen sequences. A degenerate oligonucleotide complementary to selected mRNAs and absent in both rRNA or tRNA was identified allowing for selective transcription of mRNA. To demonstrate the power of MTP, species-specific primers were designed and tested on RNA from Saccharomyces cerevisiae exposed to nitrogen deficiency, and on RNA from Neurospora crassa during early sexual development following nitrogen depletion. When primary nitrogen sources are not available or are present in concentrations low enough to limit growth, many different nitrogen sources can be used. Utilization of secondary nitrogen sources is highly regulated, and nearly always requires the synthesis of a set of pathwayspecific catabolic enzymes and permeases [15]. Several studies have shown the induction of a common suite of effector genes during growth of fungal plant pathogens under nitrogen-starved conditions in vitro and during growth in planta $[16,17]$. As a consequence, nitrogenstarved media has become a model for the environment that a pathogen encounters during growth in planta
[16]. Furthermore, nitrogen uptake and exchange are key processes for ectomycorrhizal interactions that are established between the root systems of terrestrial plants and hyphae from soil-borne fungi $[18,19]$. Finally, nitrogen deficiency has been associated with major problems encountered in contemporary wine making [20], especially those related to slow and incomplete fermentations [21]. Therefore, the response of budding yeast exposed to nitrogen starvation has been of interest in light of nutrient depletion during wine fermentation.

Neurospora crassa is a heterothallic filamentous fungus that undergoes a complex pattern of sexual differentiation to form the female reproductive structure (protoperithecium) when subjected to conditions of nitrogen starvation, light, and low temperature [22]. A large number of genes affecting sexual development have been identified by mutation $[23,24]$, but large-scale transcript profiling has not been performed.

Here we show for these applications that the addition of an MTP provides superior sensitivity and precision to microarray transcript profiling compared to sole use of oligo-dT primers. We corroborate these results for high throughput RNA tag sequencing, incorporating modification of the Illumina Digital Gene Expression protocols to facilitate use of MTPs.

\section{Results}

Transcript profiling of S. cerevisiae grown in nitrogenpoor conditions

We identified a 12-nucleotide (nt) degenerate sequence that occurs one or more times in $76 \%$ of 6608 mRNAs present in budding yeast, Saccharomyces cerevisiae, but that is absent in rRNA or tRNA. The corresponding degenerate primer was NDKTBBBBDWGS. Among the 5039 yeast ORFs containing the identified degenerate sequence, $86 \%$ featured one to five exact priming sites and the remaining sequences featured six to 44 priming sites (see Additional File 1). On average, there were 3.2 priming sites per gene. Priming for reverse transcription is not highly specific [8], thus MTPs may prime nearly all genes with some frequency. Nonetheless, the MTP and an equal proportion of oligo-dT primers were supplied for the reverse transcription step during the microarray target labeling procedure, to achieve the greatest coverage possible.

Contrasting yeast grown in nitrogen-poor and nitrogen-rich conditions, priming by MTP increased number of well measured genes, recorded after data normalization, by $31 \%$ over oligo-dT priming (Table 1; see Additional File 2). A logistic regression indicates that a model featuring the number of MTP binding sites in a gene is a statistically significantly better predictor of whether a statistically significant difference in expression will be observed when using MTPs than is a model 
Table 1 Overview of the results comparing use of oligo(dT) and multi-targeted primers (MTPs) for reverse transcription

\begin{tabular}{|c|c|c|c|c|c|c|}
\hline \multirow[t]{2}{*}{ Feature } & \multicolumn{3}{|c|}{$\begin{array}{c}\text { Saccharomyces cerevisiae } \\
\text { Nitrogen depletion }\end{array}$} & \multicolumn{3}{|c|}{$\begin{array}{c}\text { Neurospora crassa } \\
\text { Protoperithecial developmenr }\end{array}$} \\
\hline & Oligo (dT) & MTP & Common & Oligo (dT) & MTP & Common \\
\hline Number of well measured genes ${ }^{1}$ on the array & 4620 & 6042 & 4573 & 469 & & 416 \\
\hline Number of genes significantly differentially expressed ( $P \leq 0.05)$ & 2155 & 3577 & 1839 & 172 & 406 & 2021 \\
\hline Number of up-regulated genes $(P \leq 0.05)$ & 1080 & 1855 & 925 & 66 & 232 & 31 \\
\hline Number of down-regulated genes $(P \leq 0.05)$ & 1075 & 1722 & 912 & 106 & 174 & 61 \\
\hline Highest gene expression ratio & 49.15 & 32.6 & & 24.26 & 10.74 & \\
\hline Average gene expression ratio & 2.05 & 1.77 & & 1.96 & 1.43 & \\
\hline $\mathrm{GEL}_{50}^{2}$ & 1.51 & 1.27 & & 1.91 & 2.08 & \\
\hline Number of genes with gene expression ratio greater or equal to GEL50 $(P \leq 0.05)$ & 1237 & 2617 & 1166 & 102 & 141 & 52 \\
\hline
\end{tabular}

${ }^{1}$ well measured genes recorded after data normalization (foreground fluorescence signals were at least three standard deviations of the distribution of intensities of the background pixels for that gene).

${ }^{2} \mathrm{GEL}_{50}$ - gene expression level at which there is a $50 \%$ empirical probability of a significant call.

without a slope with regard to number of MTP binding sites $(\mathrm{P}<0.0001$, slope 0.05$)$. However, a model featuring the number of MTP binding sites is also a statistically significant predictor of the probability of a statistically significant call when oligo-dT alone is used $(P=0.02$, slope 0.03). More saliently, use of the MTP increased the number of genes that were identified as significantly differentially expressed by $66 \%$.

We analyzed the frequency of MTP priming sites among genes identified as significantly differentially expressed in nitrogen-depleted yeast. Out of 1738 genes indicated by MTPs exclusively as differentially expressed, 342 did not have an MTP recognition site and 1396 genes contained one to 29 priming sites. Among 316 genes exclusively identified by oligo-dT primers as significantly differentially expressed in response to nitrogen depletion, 68 did not contain an MTP recognition site and 248 exhibited one to 18 priming sites. For the genes with the highest number of priming sites, the gene expression levels calculated based on the MTP experiment were similar to the oligo-dT experiment and typically yielded ratios close to one, indicating that a higher number of exact MTP priming sites per se did not increase detection of significant gene expression differences.

The ratio of genes abundantly expressed to genes meagerly expressed was largely unchanged when MTPs were used (Table 1). Just two genes manifested a statistically significant opposite direction of differential expression in the MTP-microarray dataset compared to both the oligo-dT dataset and the independent RNA-Seq datasets (YDR179W-A, YKL203C). Out of 3577 genes detected as significantly differentially expressed by MTP-microarray, this is a very small number and may be reasonably attributed to experimental error in the MTP-primed reverse transcription and hybridizations. The highest and average changes in gene expression level were lower for MTP than for oligo-dT (Table 1). Lastly, the gene expression level at which there was a $50 \%$ empirical probability of a significant call $\left(\mathrm{GEL}_{50}\right)$, illustrating the statistical power of the experiment, demonstrated greater resolution for MTP than for oligo$\mathrm{dT}$ primers (Table 1). Three times more genes were detected as differentially expressed in the MTP experiment above its GEL $_{50}$ threshold than were differentially expressed above the $\mathrm{GEL}_{50}$ threshold of the experiment performed with oligo-dT primers alone (Table 1).

Functional classification of genes differentially expressed by $S$. cerevisiae grown in nitrogen-poor conditions While meager expression in nitrogen-poor medium was typical for genes functioning in protein synthesis and transcription, abundant expression was typical for genes coding for proteins involved in energy and genes of unknown function (Figure 1a). This result was obtained both with the aid of MTPs and with oligo-dT primers. Using oligo-dT primers, functional categories of metabolism, development, and energy were identified as significantly affected under nitrogen deficiency (Fisher's Exact test, $P=0.01, P=2.3 \times 10^{-21}, P=0.01$ ). The MTP experiment identified the same functional categories, and provided additional experimental power, also identifying genes involved in regulation with the environment $\left(P=4.9 \times 10^{-5}\right)$. The classification for which the highest number of genes was indicated as significantly differentially expressed by either approach was metabolism. Within metabolism, the greater power of the MTP experiment provided a stronger signal of subcategory, as more measurements were statistically significant: $39 \%$ as indicated by MTP and $15 \%$ as indicated by oligo-dT primers were involved in amino acid metabolism; $36 \%$ and $15 \%$, respectively, in carbohydrate metabolism; and $16 \%$ and $6 \%$ in lipid metabolism. 


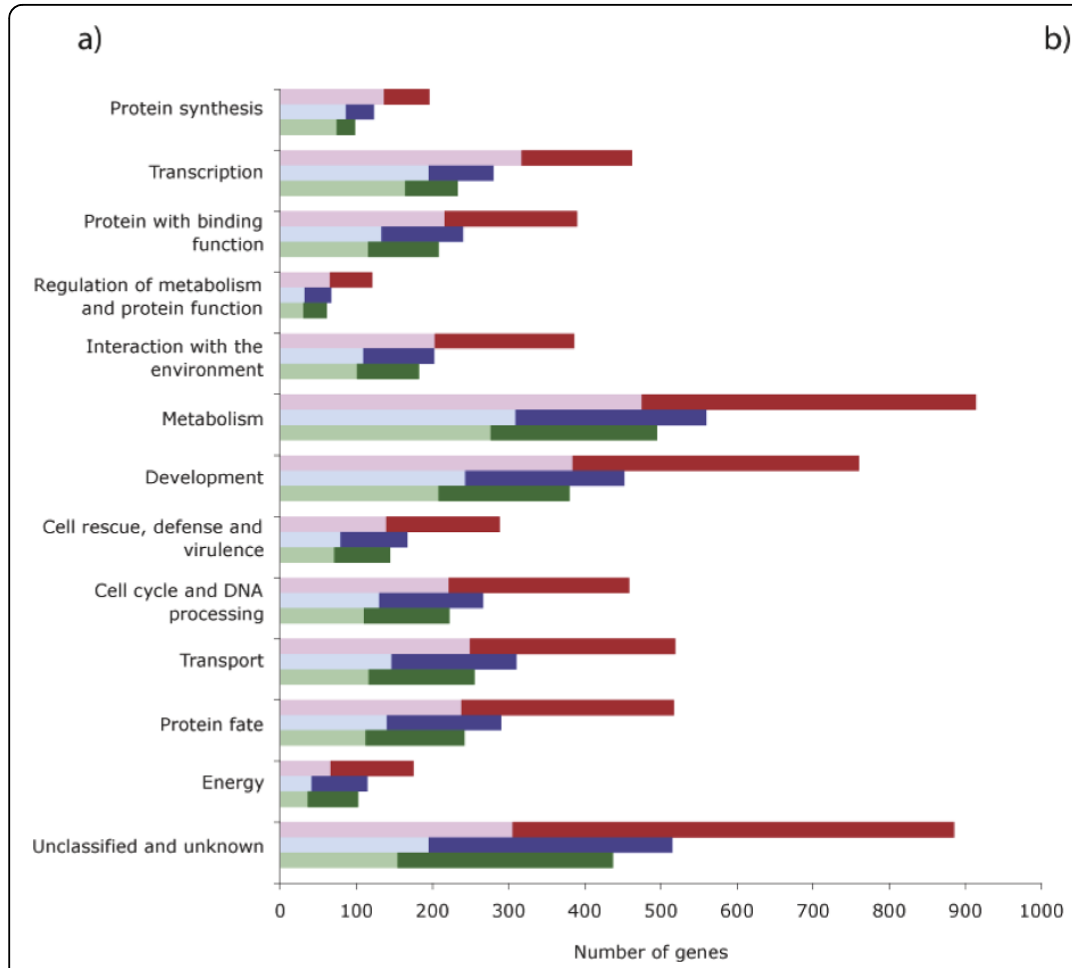

b)

Figure 1 Functional classification of genes significantly differentially expressed $(P \leq 0.05)$ by a) $S$. cerevisiae grown in nitrogen-poor conditions and b) $\boldsymbol{N}$. crassa protoperithecia identified by transcript profiling using oligo(dT) (red) or multi-targeted primers (blue). Genes identified by both methods marked in green. Light-colored bars represent genes meagerly expressed and dark-colored bars represent genes abundantly expressed. The categories are sorted by the proportion of genes meagerly- to abundantly- expressed.

MTPs were not biased for inference in particular pathways or processes, providing an even increase in power across all of them. For nearly every pathway or process, the microarrays performed with MTPs resulted in a more complete assessment of expression of proteins/ enzymes building a pathway, assigning expression to more genes than the oligo-dT-primed experiment in each process (Table 2; see Additional File 3). Most of the genes coding for enzymes belonging to 26 pathways specifically related to nitrogen and amino acid metabolism and biosynthesis were meagerly expressed by yeast growing in nitrogen-poor conditions, e.g. glutamate metabolism and urea cycle (Table 2, Figures 2, 3). An opposite tendency of abundant expression in nitrogenpoor conditions was observed among genes encoding proteins composing energy-related pathways, e.g. the citrate cycle (Figures 1 \&4). Typically, enzymes catalyzing reactions occurring in opposite directions exhibited opposite regulation (Figure 2). Furthermore, we were able to more reliably characterize the expression of multiple genes coding for isozymes, isoforms with different cellular location and enzyme subunits (Figures 2, 3, 4). A total of 561 genes identified as differentially expressed by the MTP experiment and 364 genes identified as differentially expressed by the oligo-dT experiment belonged to environmental stress response genes that were inferred by evaluating the transcriptional responses to a wide range of stress stimuli, including nitrogen depletion [25].

\section{RNA sequencing for assessment of transcript abundance} in S. cerevisiae under nitrogen deficiency

To validate the microarray profiling results, we performed RNA tag sequencing with a modified Digital Gene Expression protocol that facilitated use of our custom oligonucleotide primers. We sequenced $6.0 \times 10^{5} 16-17 \mathrm{bp}$ tags for yeast grown in nitrogen-rich conditions, primed with oligo-dT, out of which $2.7 \times 10^{5}$ exactly matched a single mRNA sequence in the $S$. cerevisiae genome. Similarly, we sequenced $1.3 \times 10^{6}$ tags from nitrogen deprived yeast, primed with oligo-dT, including $5.7 \times 10^{5}$ tags with an exact single match. We sequenced $3.8 \times 10^{6}$ and $1.3 \times 10^{6}$ tags, respectively, from yeast grown in nitrogen-rich conditions that had been MTP-primed; and $3.1 \times 10^{6}$ and $1.4 \times$ $10^{6}$, respectively, for yeast deprived of nitrogen that had been MTP-primed. The MTP-primed sequencing yielded larger numbers of reads in all categories. The proportion of reads mapping to rRNAs and tRNAs was slightly increased with MTPs, but with both oligo-dT and MTP priming this proportion was negligible $(<0.01 \%$ of all single matches, Table 3). MTPs also did not prime a less 
Table 2 Comparison of oligo(dT) and MTP priming in terms of number of genes significantly differentially expressed in specific biological processes and metabolic pathways with the highest number of genes significantly differentially expressed $(P \leq 0.05)$ in $S$. cerevisiae and $N$. crassa

\begin{tabular}{|c|c|c|c|c|}
\hline Process/pathway* & Oligo(dT) & MTP & Additional genes identified by MTP & Improvement (\%) \\
\hline \multicolumn{5}{|c|}{ Saccharomyces cerevisiae, nitrogen depletion } \\
\hline Carbohydrate Metabolism & 136 & 208 & 72 & 53 \\
\hline Amino Acid Metabolism & 114 & 181 & 67 & 59 \\
\hline Lipid Metabolism & 55 & 89 & 34 & 62 \\
\hline Metabolism of Cofactors and Vitamins & 43 & 67 & 24 & 56 \\
\hline Energy Metabolism & 37 & 62 & 25 & 68 \\
\hline Glycan Biosynthesis and Metabolism & 28 & 58 & 30 & 107 \\
\hline Xenobiotics Biodegradation and Metabolism & 28 & 52 & 24 & 86 \\
\hline Nucleotide Metabolism & 31 & 49 & 18 & 58 \\
\hline Signalling & 19 & 39 & 20 & 105 \\
\hline Metabolism of Other Amino Acids & 20 & 36 & 16 & 80 \\
\hline Cell cycle & 20 & 32 & 12 & 60 \\
\hline Biosynthesis of Secondary Metabolites & 9 & 15 & 6 & 67 \\
\hline Folding, Sorting and Degradation & 7 & 12 & 5 & 71 \\
\hline Replication and repair & 8 & 8 & 0 & 0 \\
\hline Translation & 2 & 2 & 0 & 0 \\
\hline Transcription & 1 & 1 & 0 & 0 \\
\hline \multicolumn{5}{|c|}{ Neurospora crassa, protoperithecial development } \\
\hline Carbohydrate metabolism & 23 & 30 & 7 & 30 \\
\hline Translation & 25 & 29 & 4 & 16 \\
\hline Amino acid metabolism & 14 & 26 & 12 & 86 \\
\hline Lipid metabolism & 8 & 19 & 11 & 138 \\
\hline Xenobiotics biodegradation and metabolism & 5 & 17 & 12 & 240 \\
\hline Metabolism of other amino acids & 3 & 11 & 8 & 267 \\
\hline Metabolism of cofactors and vitamins & 3 & 10 & 7 & 233 \\
\hline Energy metabolism & 9 & 8 & -1 & -11 \\
\hline Glycan biosynthesis and metabolism & 0 & 5 & 5 & ** \\
\hline Nucleotide metabolism & 1 & 4 & 3 & 300 \\
\hline Biosynthesis of secondary metabolites & 2 & 3 & 1 & 50 \\
\hline Signalling & 1 & 1 & 0 & 0 \\
\hline Protein fate & 0 & 1 & 1 & ** \\
\hline Transcription & 0 & 1 & 1 & $* *$ \\
\hline
\end{tabular}

* The pathways and processes were identified using Kyoto Encyclopedia of Genes and Genomes (KEGG). For full list of S. cerevisiae pathways, see Additional File

** biological process not identified by oligo(dT) primers as including any genes significantly differentially expressed by $N$. crassa protoperithecia.

complex pool of mRNAs than do oligo-dTs. The coefficients of variation in number of tags across genes for the oligo-dT runs were 4.3 and 3.0 , and the coefficients of variation for number of tags across genes for the MTP runs were 3.3 and 3.9, so that the pool of transcriptomic tag sequences returned when priming with MTPs were no more rarified than the pool of transcriptomic tag sequences returned when priming with oligo-dT.

We identified a higher number of well-measured genes for the MTP-based dataset than for the oligo-dT data (Table 4, see Additional File 4). In comparison to the significantly differentially expressed gene list from microarray profiling of yeast using oligo-dT primers, tag sequencing confirmed $35 \%$ of the genes with gene expression levels consistent between the two methods of measuring transcript abundance, but left $57 \%$ of the genes without statistically significant confirmation ( $c f$. Figures 2, 3, see Additional File 5). In comparison to the MTP-primed microarray experiment, MTP-based tag sequencing confirmed $41 \%$ of the results, leaving $48 \%$ without statistically significant confirmation (Figures 2, 3, 4, see Additional File 5). Differences in the functional category of metabolism were validated. In particular, MTP identified 13 out of 24 mitochondrially encoded genes as statistically significantly differentially expressed. Oligo-dT identified none. Oligo-dT priming led to fewer than six tags in both conditions for 22 out of 24 mitochondrial ORFs. In contrast, with MTP, only nine ORFs show fewer than six tags. 


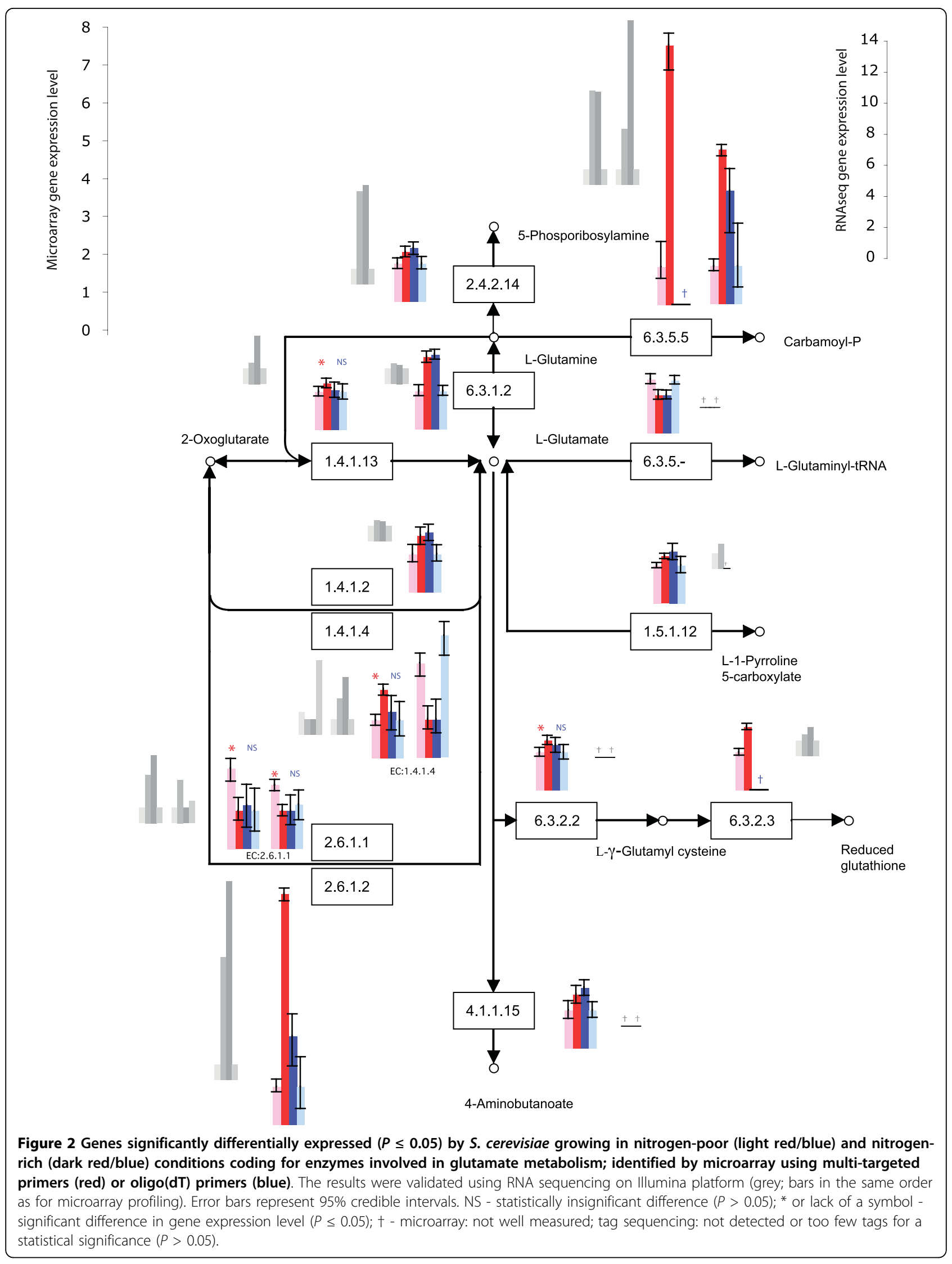




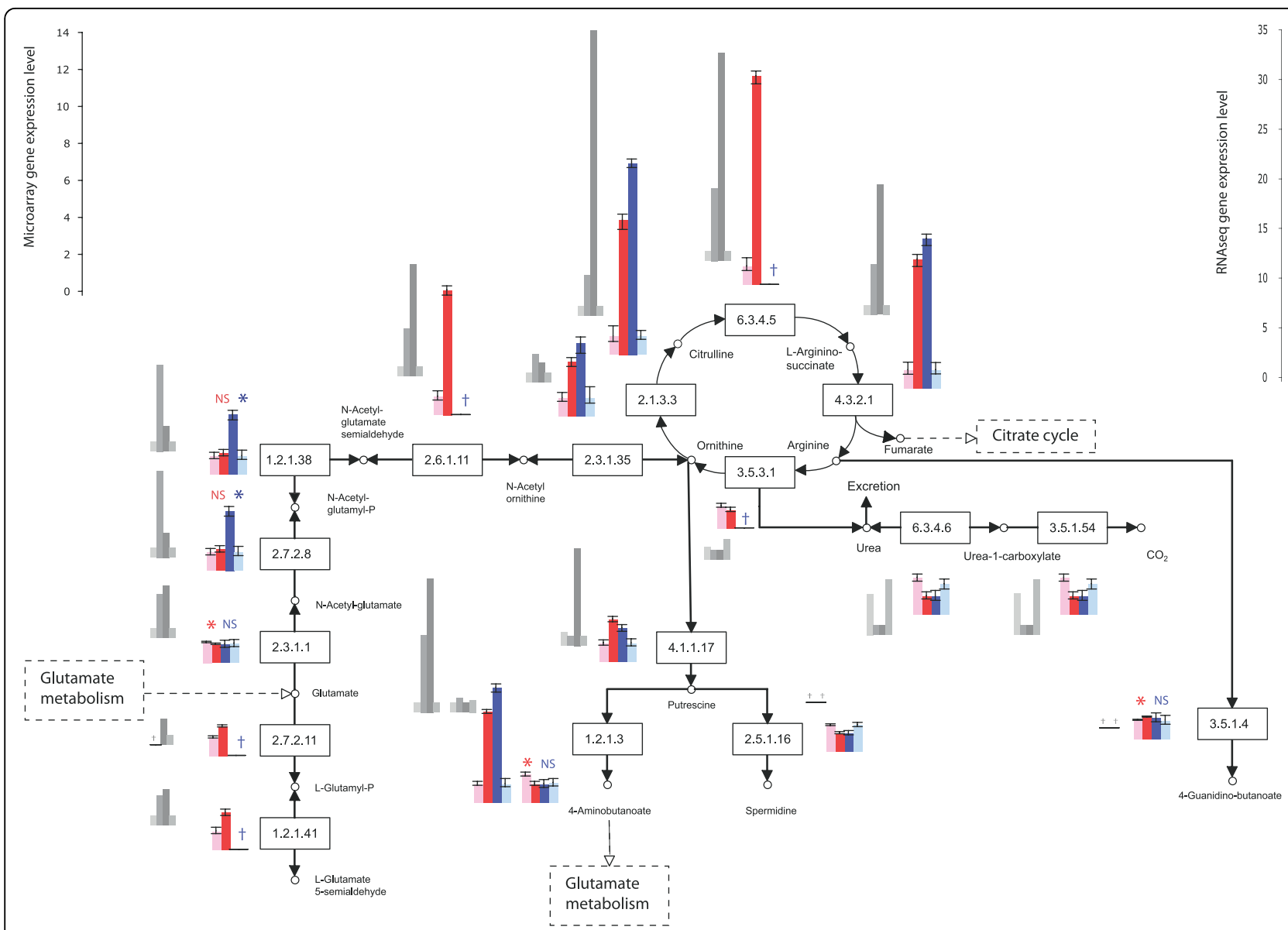

Figure 3 Genes significantly differentially expressed $(P \leq 0.05)$ by $S$. cerevisiae growing in nitrogen-poor (light colored bar) and nitrogen-rich (dark colored bar) conditions coding for enzymes involved in urea cycle and metabolism of amino groups; identified by microarray using multi-targeted primers (red) or oligo(dT) primers (blue). The results were validated using RNA sequencing on Illumina platform (grey; bars in the same order as for microarray profiling). Error bars represent $95 \%$ credible intervals. NS - statistically insignificant difference $(P>0.05) ;{ }^{*}$ or lack of a symbol - significant difference $(P \leq 0.05)$; $\dagger$ - not well measured.

Furthermore, of those nine, five are considered dubious ORFs anyway (see Additional File 4).

\section{Quantitative RT PCR validation of microarray profiling and tag sequencing of yeast grown in nitrogen-poor conditions}

To validate results of microarray and RNA sequencing using MTP, quantitative reverse transcription PCR (qRT-PCR) was performed (Table 5). Five genes were selected that were well measured by the microarrayMTP dataset but that did not produce enough signal in the microarray-oligo-dT dataset to be measured, and that showed the largest differences in expression between samples. In all five of these genes, differential expression in the direction predicted by the microarrayMTP dataset and the RNA-seq dataset was validated. Five genes were also selected that showed the largest, statistically significant differences in the dataset of microarrays primed with MTPs, but that due to lower power showed no statistically significant difference in the dataset of microarrays primed using oligo-dT. In four of these five genes, differential expression in the direction predicted by the microarray-MTP dataset and the RNAseq dataset was confirmed. In the one exception, the $\mathrm{qRT}-\mathrm{PCR}$ result contradicts the directional trend of all four previous measurements (microarrayMTP, microarray-oligo-dT, RNAseq-MTP, and RNAseqoligo-dT). Genes that were well measured in both array experiments but that were statistically significant due to the increased power of the MTP-based profiling had generally exhibited fairly low fold changes. Similarly low differences in expression in the same direction were detected by qRT-PCR, confirming the validity of MTP approach.

\section{Microarray mRNA profiling of Neurospora crassa undergoing sexual development}

The MTP for the $N$. crassa was designed targeting 9846 protein-coding ORFs. Sequence VWNVNNBDKGGC 


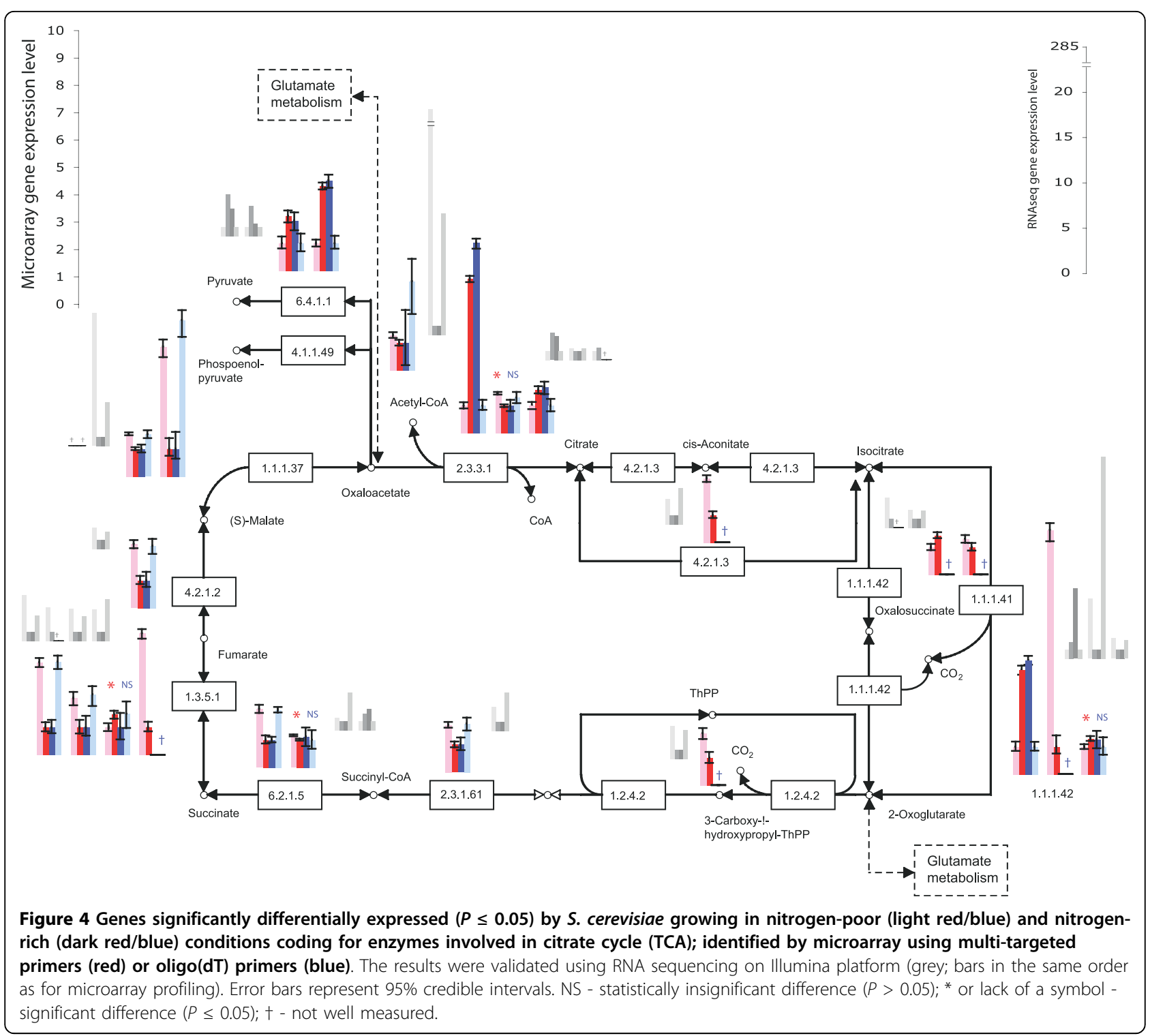

was reverse complementary to 12 -nt sequences found one or more times in $85 \%$ of the predicted mRNAs, one time in one tRNA, and one time in one rRNA. The MTP was incorporated into an experiment to quantify gene expression in mycelia and protoperithecia formed by the fungus during growth on nitrogen-poor medium, and the results were compared with transcript profiling using solely oligo-dT-based reverse transcription. Application of multi-targeted primers increased the number of well-measured genes by $331 \%$ (Table 1, see Additional File 6). The number of genes identified as significantly differentially expressed in $N$. crassa protoperithecia rose by $136 \%$. The highest and the average gene expression ratio estimated based on the MTPbased experiment were lower than for data obtained with only oligo-dT primers (Table 1 ). The $\mathrm{GEL}_{50}$ value was similar when MTPs were included, compared to when only oligo-dT primers were included. However, $38 \%$ more genes were detected as differentially expressed in the MTP experiment above the GEL 50 threshold than were differentially expressed above the $\mathrm{GEL}_{50}$ threshold in the experiment primed with oligo-dT primers alone (Table 1). Thus, more gene expression levels were precisely estimated with MTPs, even though in this case there was not a finer level of resolution for small changes in gene expression.

\section{Functional classification of genes significantly} differentially expressed by $\boldsymbol{N}$. crassa protoperithecia

Within each of the functional groups of protein synthesis, transcription, energy, and genes of unknown function, a higher proportion of genes were meagerly 
Table 3 Overview of transcriptomic sequence reads, comparing numbers of matches to coding ORFs, rRNAs, and tRNAs

\begin{tabular}{|c|c|c|c|c|c|}
\hline & Single match mRNA & Single match rRNA & Single match tRNA & Multiple matches & Unmatched \\
\hline Oligo-dT N-rich & 266258 & 40 & 18 & 72849 & 258323 \\
\hline Oligo-dT N-poor & 569310 & 38 & 12 & 99222 & 673334 \\
\hline MTP N-rich & 1286862 & 438 & 18 & 1197105 & 1312253 \\
\hline MTP N-poor & 1444282 & 1418 & 21 & 470558 & 1136358 \\
\hline
\end{tabular}

expressed in N. crassa protoperithecia than were abundantly expressed (Figure 1b). While the functional classifications with the highest number of genes significantly differentially expressed in protoperithecia were the "unknown" functional category or were metabolismrelated, the oligo-dT-primed experiment indicated that genes involved in protein synthesis were significantly affected by sexual development (Fisher's Exact test, $P=$ $\left.2 \times 10^{-6}\right)$. Application of MTPs for $N$. crassa microarray profiling additionally identified the functional categories of metabolism $(P=0.003)$ and energy $(P=0.03)$ as including a higher proportion of genes differentially expressed in protoperithecia. Enzymes involved in fatty acid metabolism were frequently abundantly expressed in $N$. crassa protoperithecia as compared to mycelia (Figure 5), while genes coding for ribosomal proteins were generally meagerly expressed (see Additional File 7). Microarray hybridizations performed with MTPbased reverse transcription identified 1 to 12 more genes involved in each biological process than did microarrays performed using oligo-dT primers only (Table 2). MTPs outperformed oligo-dT primers most strikingly in their ability to reveal differentially expressed genes in the category of unknown and unclassified proteins (Figure 1b). A gene involved in control of sexual development, encoding clock controlled pheromone $c c g$ 4 precursor, was among genes differentially expressed in N. crassa protoperithecia (see Additional File 6).

Table 4 Overview of differential expression from transcriptomic tag sequencing, comparing results of oligo-dT and multi-targeted primers (MTPs) for reverse transcription

\begin{tabular}{lccc}
\hline \multirow{2}{*}{ Feature } & \multicolumn{3}{c}{$\begin{array}{c}\text { Saccharomyces cerevisiae } \\
\text { Nitrogen depletion }\end{array}$} \\
\cline { 2 - 5 } & $\begin{array}{c}\text { Oligo } \\
\text { (dT) }\end{array}$ & MTP & Common \\
\hline Recorded genes & 4858 & 5078 & 4710 \\
Genes significantly differentially expressed & 2272 & 2861 & 1437 \\
$(P \leq 0.05)$ & 958 & 1437 & 522 \\
Up-regulated genes $(P \leq 0.05)$ & 1314 & 1427 & 633 \\
Down-regulated genes $(P \leq 0.05)$ & 166 & 284 & \\
Highest gene expression ratio & 1.84 & 1.90 & \\
Average gene expression ratio & \multicolumn{3}{c}{}
\end{tabular}

\section{Discussion}

Our multi-targeted priming method features selective targeting of multiple genomic sequences and counterselection against undesirable sequences. For genomewide gene expression assays, the addition of multitargeted primers offers the potential for improving the coverage of the reverse transcription. Reverse transcription reactions are fairly nonspecific: transcription may be primed by non-complementary primers, other RNA molecules present in the sample, and even by dNTPs [8], so the priming strategy selected may be expected to play a significant role in determining the results obtained. For instance, commonly-used oligo-dT primers generate a high frequency of truncated cDNAs through internal poly-A priming [26]. We demonstrated that addition of MTP to commonly used oligo-dT primers for yeast and $N$. crassa microarray profiling substantially increased the number of well measured genes recorded after data normalization as well as the number of genes significantly differentially expressed, compared with the sole use of oligo-dT primers (Table 1). Additionally, MTP-based profiling manifested higher statistical power as demonstrated by lower value of $\mathrm{GEL}_{50}$, a measure of empirical power to reveal differences in gene expression [27], in the S. cerevisiae experiment.

High-throughput RNA tag sequencing validated our findings. It confirmed the utility of MTPs, combined with oligo-dT column purification, for priming reverse transcription reactions, yielding an increased number of detected and well measured genes, compared to oligodT primers alone (Table 3). Priming using MTPs, we were able to corroborate gene expression levels for a higher number of genes than when priming with oligodT (41\% and 35\%, respectively; Figures 2, 3, 4). To explain this result, note that both traditional oligo-dT and MTP priming provide biased counts and profile only a proportion of the total RNA pool. Some genes that are poorly primed by oligo-dT primers are those that have protected poly-A tails due to foldback structures; other genes, such as histones in metazoan DNA, have no poly-A tail. In a particuliarly clear case for yeast, it has been demonstrated that many to all of the 24 putative and known mitochondrial mRNAs are not stabilized by a poly-A tail [28-31]. For these genes, MTPs were successful at priming and revealing gene 
Table 5 Validation of microarray and RNA sequencing of yeast grown in nitrogen poor (MM) and nitrogen rich (SC) conditions using Real Time RT PCR

\begin{tabular}{|c|c|c|c|c|c|c|c|c|c|c|}
\hline \multirow{3}{*}{ Gene ID } & \multicolumn{4}{|c|}{ Microarray } & \multicolumn{4}{|c|}{ RNA sequencing } & \multicolumn{2}{|c|}{ RT PCR } \\
\hline & \multicolumn{2}{|l|}{ MTP } & \multicolumn{2}{|c|}{ oligo(dT) } & \multicolumn{2}{|l|}{ MTP } & \multicolumn{2}{|c|}{ oligo(dT) } & \multirow{2}{*}{$\begin{array}{c}\text { MTP } \\
\text { Ratio MM/SC }\end{array}$} & \multirow{2}{*}{$\begin{array}{c}\text { Oligo(dT) } \\
\text { Ratio } \mathrm{MM} / \mathrm{SC}\end{array}$} \\
\hline & Ratio MM/SC & $p$ value & Ratio MM/SC & $p$ value & Ratio MM/SC & $p$ value & Ratio MM/SC & $\mathrm{p}$ value & & \\
\hline YDL244W & 1.98 & 0.00 & & & 3.16 & 0.11 & & & 28.94 & 39.62 \\
\hline YGR225W & 1.72 & 0.00 & & & 7.65 & 0.00 & & & 4.08 & 3.13 \\
\hline YGR249W & 1.66 & 0.01 & & & 5.80 & 0.00 & & & 4.79 & 8.20 \\
\hline YMR025W & 1.51 & 0.00 & & & 3.96 & 0.15 & & & 2.10 & 1.57 \\
\hline YNR072W & -1.24 & 0.05 & & & -6.32 & 0.00 & & & -2.34 & -1.36 \\
\hline YFR034C & -1.42 & 0.02 & -1.25 & 0.10 & -9.08 & 0.00 & -6.41 & 0.08 & 1.30 & 1.24 \\
\hline YKR053C & -5.37 & 0.00 & -1.88 & 0.14 & -2.16 & 0.00 & 1.15 & 0.51 & -2.10 & -2.05 \\
\hline YLR126C & 1.54 & 0.00 & 1.36 & 0.11 & 5.54 & 0.05 & 1.40 & 0.42 & 1.58 & 1.95 \\
\hline YMR003W & 1.25 & 0.01 & 1.11 & 0.26 & 7.91 & 0.01 & 2.65 & 0.08 & 1.03 & 1.95 \\
\hline YOR252W & 1.29 & 0.01 & 1.03 & 0.46 & 6.33 & 0.00 & 1.54 & 0.10 & 1.99 & 1.54 \\
\hline
\end{tabular}

expression differences, whereas oligo-dT primers were not. Some genes feature multiple sites for priming by MTPs, leading to greater power for the detection of differential expression for those genes. However, the effect of multiple priming is modest, mostly increasing the chance of a gene being well measured. The equivalent coefficients of variation of our transcriptomic tag sequencing runs indicate that the complexity of the mRNA pool with either priming strategy is approximately the same.
The advantage of adding MTPs obtains because the reverse transcription biases for each method of priming are different. In combination, they exhibit greater coverage than either would individually. Based on our highthroughput transcript sequencing data, MTPs might yield slightly better coverage of expressed sequences than oligo-dT. In terms of false negatives in the high-throughput transcript sequencing, MTP priming exhibited a lower false-negative rate (248 not detected by MTP mix

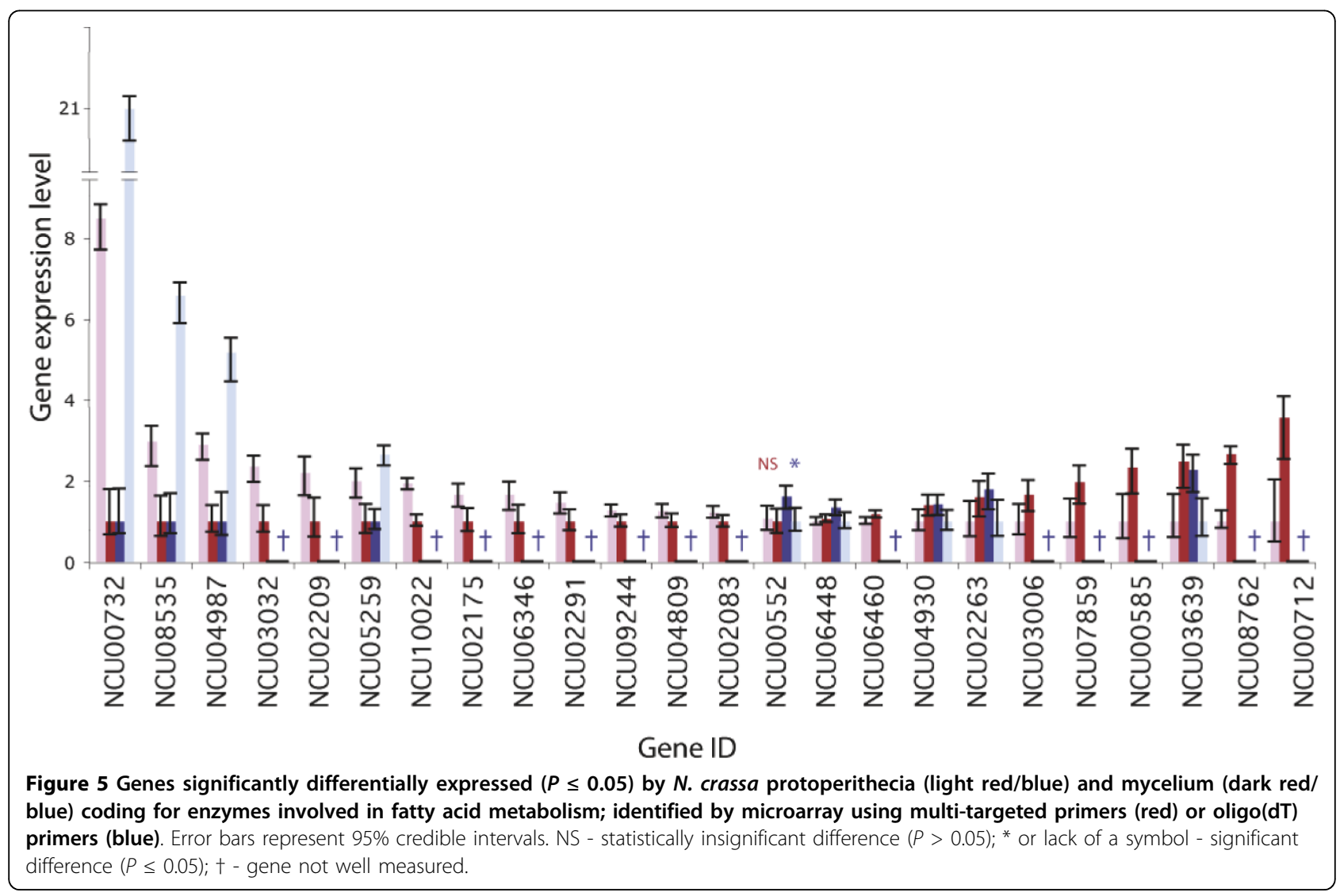


that were detected by oligo-dT alone) than did oligo-dT priming alone (368 not detected by oligo-dT that were detected by MTP). Correlations between replicate tag sequencing results and between replicate MTP-based microarray results were similar. Next generation RNA tag sequencing offers a promise of increasing power, depth, and sensitivity of gene expression analysis, but even a limited depth of RNA sequencing remains costly compared to a highly replicated experiment using microarrays generated in the lab.

Finally, qRT-PCR validated the results based on MTPpriming. It confirmed that genes identified as well measured or as significantly differentially expressed only by MTP-primed microarray, had changed in expression in the direction revealed by the MTP-primed experiments, regardless of the primer combination used. This observation suggests that incorporating MTPs increases the power for detection of differential expression by microarrays, by increasing the coverage of the priming of reverse transcription while maintaining low noise due to nonspecific priming. Logistic regressions showed some evidence that genes with more priming sites were measured with increased power with MTPs applied than genes with fewer MTP priming sites, but this observation was true (albeit to a lesser extent) for oligo-dT priming as well. This commonality may well arise because longer genes have more MTP sites and are better measured with either MTPs or oligo-dT primers. Considering the 4762 observations underlying these effects, their statistical significance is unsurprising. The effect sizes are not large. It would be possible to procedurally minimize the variation in the number of MTP sites, but effectively, this minimization of variation would entail searching for MTPs that were less powerful for some genes. That would be counter-productive to the effort to measure genome-wide gene expression for as many genes as possible to the best precision possible. Because such gene-specific effects exist at approximately the same levels no matter the method of priming, decent statistical approaches toward microarray data have featured effects estimated for each gene, thus providing $\mathrm{P}$-values and confidence or credible intervals that appropriately reflect the variation in power across genes. Markedly successful enhancement of the power of transcriptomic approaches was achieved here by usage of MTPs subsequent to a crude filtration provided by poly$\mathrm{A}+$ purification columns, which typically bring the RNA content to approximately $50 \%$ poly-A+ RNA and $50 \%$ other RNA $[32,33]$. An alternate approach with potential to further increase power would be to forego any column purification of poly-A+, relying solely on MTPs to pick out messenger RNA from other species of RNA.

Functionally, inclusion of MTPs provided a more complete picture of cellular processes undergoing change during fungal growth in nitrogen deficient conditions and in early sexual development. Interestingly, the most striking difference in the ability to detect genes differentially expressed between MTPs and oligo-dT primers was observed among $N$. crassa unknown and unclassified genes (Figure 1b). Genes expressed at a low level have a lower probability of being identified and characterized than those expressed at a higher level [34]. Only $42 \%$ of genes in $N$. crassa genome have been assigned known functions [35] so identifying their expression signatures and inferring functional classification are key objectives of future experimentation [36]. The increased levels of yeast transcripts coding for enzymes involved in the TCA cycle observed here (Figure 4) supports a finding that the content of TCA cycle compounds increases during yeast starvation [37]. Both carbon and nitrogen limitation have been shown to enhance respiration relative to fermentation in yeast $[21,38]$. Amino acid metabolism was strongly affected by exposure to nitrogen deficiency, as in the filamentous fungus Magnaporthe grisea [17]. Prevalent down-regulation of glutamate metabolism pathway (Figure 2) was in accordance with decline of total glutathione pool found in nitrogen-starved S. cerevisiae [39]. Similarly, the urea cycle was down-regulated, with an exception of the branch responsible for urea degradation (Figure 3). Urea amidolyase, the enzyme corresponding to that branch, contains both urea carboxylase and allophanate hydrolase activities. It also converts allantoin to ammonia and carbon dioxide, enabling S. cerevisiae to use allantoin as a sole nitrogen source [40]. The urea cycle operates in terrestrial animals to detoxify ammonia, and in yeast plays a principal role in the biosynthesis of arginine. Within these pathways, we were able to characterize expression of multiple isozymes and isoforms (Figures 2, 3,4 ) providing a key component of a full understanding of expression regulation in nitrogen-deficient medium. The general trend of shutting down amino acid biosynthesis and metabolism appears essential for survival during prolonged periods of starvation. These results from our experiments are entirely consistent with a functional category analysis of independent data from the unreplicated time-course experiment by Gasch et al. [25].

A number of genes abundantly expressed by yeast during growth in nitrogen-poor conditions are components of the environmental stress response that was characterized by evaluating the transcriptional responses to a wide range of stress stimuli, including nitrogen depletion [25]. The remaining genes might be involved in immediate responses specific to nitrogen limitation, but many are likely associated with transitive effects that occur over time, such as successive nutrient depletion, $\mathrm{pH}$ change, cell density, and growth arrest. The finding that development-related genes were significantly 
affected in our study may be related to growth arrest or to a morphological switch from the yeast to a filamentous or pseudohyphal growth triggered by nitrogen starvation in the presence of a fermentable carbon source, such as glucose [41]. Nutrient and nitrogen sensing plays important roles in fungal development in general, and specifically in critical aspects of pathogenicity and virulence, for both animal and plant pathogens. Dimorphic pathogens such as the phytopathogenic smut fungi, Ustilago maydis and Microbotryum violaceum, must switch from a yeast-like to a filamentous form in order to cause disease [42].

$N$. crassa protoperithecia consist of a small knot of vegetative hyphae surrounding ascogonial cells. Their formation requires extensive cell proliferation. Thus, it was not surprising to find a large proportion of differentially expressed genes involved in metabolism, especially carbohydrate metabolism (Table 2), providing energy and the robust structural components necessary for specialized cell formation. The shift in transcript accumulation of genes involved in fatty acid metabolism (Figure 5) supports previous observations that the fatty acid composition of sexual tissues of Neurospora differ substantially from the composition of asexual tissues. For instance, mutations in the gene encoding the b-fatty acid synthase affect sexual development [43]. On the other hand, we expected to identify known developmental genes responsible for the formation of protoperithecial reproductive structures, but the number of genes significantly differentially expressed involved in the functional category of development was very low. One of them coded for clock controlled pheromone $c c g-4$ precursor which had been first identified as a gene that is expressed with a $22 \mathrm{~h}$ rhythm under the control of the circadian biological clock [44]. The expression of the pheromone precursor genes is mating-type specific and is under the control of the mating type locus. These genes are highly expressed in conidia and under conditions that favor sexual development [45].

We found a reduced level of transcripts related to protein synthesis (Figure 1, see Additional File 7) in both $S$. cerevisiae under nitrogen starvation and in $N$. crassa sexual development occurring as a developmental response to nitrogen depletion. Ribosome biogenesis and protein translation are among the most energy-consuming cellular processes [46]. Thus, these pathways are tightly controlled upon nutrient limitation. Decreasing levels of protein, RNA and soluble aminonitrogen were observed during protoperithecia development as early as 1975 [47]. Additionally, ribosomes are rapidly degraded by autophagy upon nutrient starvation in S. cerevisiae, implying that degradation of excessive ribosomes may help to shut down protein translation rapidly and provide an important source of new building blocks to maintain cellular homeostasis [48].

The MTPs used in this study were designed to prime $76-85 \%$ of mRNAs rather than all the genes, so that reverse transcription of rRNA and tRNA could be simultaneously minimized. An additional step of primer design could be conducted to specifically target the remaining sequences to eliminate or further diminish the need to rely on low-complexity, low-specificity oligo-dT primers. For transcript profiling in prokaryotes, such a tiered approach would constitute an appealing alternative to the use of non-specific random primers. Correspondingly, the modification of Illumina massively parallel RNA tag sequencing presented here, involving the use of custom primers, presents the possibility of performing Digital Gene Expression assays on transcriptomes lacking poly(A) tails or on a designable fraction of all the genes. Furthermore, future applications of multi-targeted primers may include concurrent selective amplification of DNA sequences of interest from one or multiple genomes for the high-throughput sequencing of multiple homologous loci. Locked nucleic acid nucleotides could be included in the MTP sequence to increase binding strength and specificity, and to decrease non-specific amplification [49].

\section{Conclusions}

Here we presented a novel priming strategy for genomewide gene expression assays, featuring selective targeting of mRNA and counter-selection against rRNA and tRNA. We demonstrated superior performance of two MTPs compared to oligo-dT by microarray profiling of the response of Saccharomyces cerevisiae to nitrogen deficiency and by profiling Neurospora crassa early sexual development. MTPs resulted in higher sensitivity, yielding more well measured genes after data normalization, more genes significantly differentially expressed, and a greater power to detect meager differences in gene expression. Our results provide the most complete and detailed expression profiles of the nitrogen starvation response to date. Future applications of multi-targeted primers may include concurrent selective amplification of DNA sequences of interest from one or multiple genomes for the high-throughput sequencing of multiple homologous loci.

\section{Methods}

\section{Multi-targeted primers (MTPs)}

Sequences for all three types of RNA were obtained from the Saccharomyces Genome Database [50] and the Neurospora crassa Genome Database [35]. To identify a degenerate sequence that occurs in a maximal number of mRNAs and in a minimal number of tRNAs and rRNAs, exhaustive search is not a feasible option as 
there are $16^{12}$ such degenerate sequences. Our heuristic search started with a random degenerate 12-mer oligonucleotide. Iteratively, this oligonucleotide was randomly mutated and a score was computed as $m /\left(m_{t} *(1+t+\right.$ $r)$ ), where $m, t$, and $r$ are the number of hits of the degenerate sequence to the annotated mRNAs, tRNAs, and rRNAs, respectively, and $m_{t}$ is the total number of annotated mRNAs. If a randomly generated number (01) was smaller than the ratio of the new score to the previous, raised to the power of ten, then the new oligonucleotide was kept. Otherwise, it was discarded. This procedure was iterated so that the oligonucleotide experienced $2 * 10^{6}$ mutations before the search was terminated. To ensure convergence upon a globally optimal priming sequence, several different random initial sequences were used.

Each new oligonucleotide obtained was recorded. From the ranked list, we selected a high-scoring sequence that additionally showed strong binding (GC) in the $3^{\prime}$ end. The MTP primer was designed to be reverse-complementary to the selected dodecanucleotide.

\section{Yeast strain and conditions}

Saccharomyces cerevisiae S288c was grown in synthetic complete medium (SC) at $30^{\circ} \mathrm{C}$ shaking at $160 \mathrm{rpm}$ until mid log phase $\left(\mathrm{OD}_{600}=0.4\right)$. For nitrogen depletion, cells were collected and resuspended in an equal volume of minimal medium without amino acids or adenine and with limiting concentrations $(0.025 \%)$ of ammonium sulfate. Cultures were harvested after $12 \mathrm{~h}$ incubation, flash frozen in liquid nitrogen and stored at $-80^{\circ} \mathrm{C}$ [25].

\section{Sample preparation and hybridization to yeast microarrays}

A set of clones containing 6,188 verified open reading frames (ORFs) from the Saccharomyces Genome Project was amplified by PCR and the DNA was spotted on CMT-GAPS $\gamma$-aminopolysilane-coated glass slides (Corning, Corning, NY). RNA was extracted and cDNA synthesized as in Townsend et al. [51]. Briefly, two $\mu \mathrm{g}$ of purified mRNA were reverse transcribed using the reverse transcriptase Superscript II (Invitrogen, Carlsbad, CA) for $2 \mathrm{~h}$ at $42 \mathrm{C}$. The reaction was primed with either $0.5 \mu \mathrm{g}$ oligo-dT primers or $0.25 \mu \mathrm{g}$ oligo-dT mixed with $0.25 \mu \mathrm{g}$ of yeast-specific MTP. Amino-allyldUTP (Sigma) was incorporated into cDNA along with dNTPs. The cDNA was labeled reciprocally with cyanine dyes and used for hybridization as Townsend et al. [51]. The cDNA from yeast cultured in nitrogen-rich and the cDNA from yeast cultured in nitrogen-depleted medium were reciprocally labeled and competitively hybridized. All replicates originated from independent reverse transcription reactions.

\section{Data acquisition and analysis}

Hybridized microarrays were scanned and gridded with a GenePix 4000B microarray scanner (Axon Instruments, Foster City, CA), normalized by background-subtracted mean-by-mean normalization of well measured spots as in [51,52], and statistically analyzed using a Bayesian analysis of gene expression levels (BAGEL [53]). Fluorescence intensity values were adjusted by subtracting background from foreground. A gene was considered well measured if the foreground fluorescence signals were higher than three standard deviations of the distribution of intensities of the background pixels for that gene. Genes were deemed significantly differentially expressed when $P \leq 0.05$. To assess the power of the experiments to detect smaller differences in gene expression, the gene expression level at which there was a $50 \%$ empirical probability of a significant call $\left(\mathrm{GEL}_{50}\right)$ was inferred by logistic regression of statistical significance against fold-change as in Townsend [27]. Functional annotation was performed based on the Saccharomyces Genome Database [50] and Kyoto Encyclopedia of Genes and Genomes (KEGG) [54]. Significance of abundantly differentially expressed functional categories was calculated using a Fisher's exact test. Raw expression data and analysis results are deposited under accession \#25 at the Filamentous Fungal Gene Expression Database (FFGED) $[36,55]$ and under accession GSE230003 at the NCBI Gene Expression Omnibus (GEO) database [56].

\section{Transcriptional tag sequencing of yeast in nitrogen-poor conditions}

Digital Gene Expression (DGE) measurement was performed using Tag Profiling with DpnII Sample Prep Kit (Illumina, San Diego, CA). Oligo-dT priming was performed as instructed using $2.5 \mu \mathrm{g}$ of the same total RNA that was extracted from S. cerevisiae for microarrays. For multi-targeted priming, mRNA was purified on Oligo-dT Cellulose Columns (Molecular Research Center, Cincinnati, $\mathrm{OH}$ ) and $2 \mu \mathrm{g}$ were used for reverse transcription with $0.5 \mu \mathrm{g} 5^{\prime}$-amine yeast-specific MTP and $5 \mathrm{mC}$ dNTP $10 \mathrm{mM}$ each (Illumina). The resulting first strand cDNA was cleaned with Microcon-30 microconcentrators and coupled to Sera-Mag ${ }^{\oplus}$ magnetic carboxylate microparticles (Seradyn, Indianapolis, IN). cDNAs was immediately subjected to second strand synthesis and sequencing library preparation with the Illumina kit. Samples were then sequenced on an Illumina Genome Analyzer II. Raw sequence reads of transcript tags are deposited under accession \#67 at the FFGED [36,55].

\section{Transcript tag analysis}

The DGE protocol used generates 16-17 nt tags ligated to adapters. RNA sequencing produced reads of $28 \mathrm{bp}$. After discarding the included adapter sequence, we 
searched for perfect matches of the tag sequence in the $S$ cerevisiae genome. Statistical significance was calculated by estimating the proportion $p$ of tags per gene by maximum likelihood (maximizing $p^{n}(1-p)^{N-n}$ where $n$ is the number of tags observed for the gene and $N$ is the total number of tags for all genes), and comparing the likelihood of the observed numbers of tags with a single maximum likelihood proportion for both samples $(p)$ to the likelihood of the observed numbers of tags with two independent maximum likelihood proportions $\left(p_{1}\right.$ and $p_{2}$, one for each sample). To estimate $P$-values, negative two times the log likelihood ratio was compared to a chi-square distribution with one degree of freedom. Statistical results for transcript tag analysis are deposited under accession \#67 at the FFGED $[36,55]$.

\section{Real Time RT-PCR validation of yeast microarray and RNA sequencing results}

To validate results of yeast microarrays and RNA sequencing, ten genes were selected whose expression was well measured in the MTP-based experiment, but not well measured or insignificant in the oligo-dT experiment. An equal amount $(2 \mu \mathrm{g})$ of mRNA, as had been used for microarray profiling, was reverse transcribed with Superscript II reverse transcriptase (Invitrogen, Carlsbad, CA) and $0.25 \mu$ g oligo-dT mixed with $0.25 \mu \mathrm{g}$ yeast-specific MTP or $0.5 \mu \mathrm{g}$ oligo-dT. Relative transcript abundance was measured using gene specific primers (see Additional File 8) and SYBR Green PCR Master Mix (Applied Biosystems, Carlsbad, CA). The reaction was run on Applied Biosystems 7500 Fast RealTime PCR System according to manufacturer's recommendations. A gene coding for mitochondrial ribosomal protein of the small subunit (YDR175C), whose expression did not change in the microarray experiment, was used as endogenous control. Transcript levels were calculated from triplicates within one plate using the comparative $C_{\mathrm{T}}\left(\Delta \Delta \mathrm{C}_{\mathrm{T}}\right)$ method (ABI application notes, Guide to Performing Relative Quantitation of Gene Expression Using Real-Time Quantitative PCR).

\section{Induction of Neurospora crassa sexual development}

Neurospora crassa (mat A) obtained from Fungal Genetics Stock Center (strain 2489) was grown on nitrogenpoor synthetic cross medium (SCM) agar plates with $1.5 \%$ sucrose as carbon source, at room temperature under natural light. The mycelium was flash frozen by application of liquid nitrogen, then harvested after 18 hours growth. Protoperithecia were similarly harvested, after 6 days.

\section{Transcript profiling of Neurospora crassa protoperithecia}

Total RNA was extracted from homogenized frozen tissue and mRNA was purified with TRI REAGENT and
Oligo-dT Cellulose Columns (Molecular Research Center). Reverse transcription, data acquisition and analysis were conducted as for $S$. cerevisiae. The cDNAs were hybridized [57] to whole-genome-spotted 70-mer $N$. crassa microarrays [58]. Samples from $N$. crassa mycelia and protoperithecia were competitively hybridized. Each experiment consisted of a dye-swap and an additional replicate hybridization. Functional classification was based on MIPS Neurospora crassa Genome Database [52] and KEGG [54]. Raw expression data and analysis results are deposited under accession \#2 at the FFGED $[36,55]$ and under accession GSE23003 at the NCBI GEO database [56].

\section{Additional material}

Additional File 1: Number of MTP priming sites in S. cerevisiae genes. Table of the number of MTP priming sites in S. cerevisiae genes Additional File 2: Microarray profiling results comparing $S$. cerevisiae grown in nitrogen rich (SC) or poor (MM) conditions using oligo(dT) and multi-targeted primers (MTPs) for reverse transcription. Table of microarray profiling results comparing S. cerevisiae grown in nitrogen rich (SC) or poor (MM) conditions using oligo(dT) and multi-targeted primers (MTPs) for reverse transcription. For each priming method for each gene, relative gene expression levels in SC and MM, the additions and subtractions for those levels to demarcate 95\% credible intervals, and the $\mathrm{p}$-value for the direction of difference observed are reported.

Additional File 3: Comparison of oligo(dT) and MTP priming in terms of number of genes significantly differentially expressed in specific biological processes and metabolic pathways. Table listing biological processes and metabolic pathways with the highest number of genes significantly differentially expressed $(P \leq 0.05)$ in $S$. cerevisiae and $N$. crassa, the number of genes differentially expressed as detected by oligo(dT)-primed and MTP-primed reverse transcription and microarray hybridization, the number of additional genes identified by MTP, and the percent improvement by MTP.

Additional File 4: Number of aligned reads recorded for each gene with oligo(dT)-primed and MTP-primed RNA tag sequencing of $S$. cerevisiae grown in nitrogen rich (SC) or poor (MM) conditions. Table listing each ORF, the number of oligo(dT) primed reads aligning from nitrogen poor (MM) conditions, the number of oligo(dT) primed reads aligning from nitrogen rich (SC) conditions, the number of multitarget primed reads aligning from nitrogen poor (MM) conditions, and the number of multi-target primed reads aligning from nitrogen rich (SC) conditions

Additional File 5: Comparison of transcript profiling of $S$. cerevisiae grown in nitrogen rich (SC) or poor (MM) medium obtained with microarray or RNA tag sequencing using MTP or oligo(dT) primers Table listing $S$. cerevisiae ORFs and results of both microarray and RNA tag sequencing by both MTP and oligo(dT) priming. Results listed for each platform and priming methodology are the fold change of MM/SC and the $p$-value.

Additional File 6: Microarray profiling results for $N$. crassa undergoing sexual development when multi-targeted primers or oligo(dT) primers were used for reverse transcription. Table listing the Broad ID and MIPS ID of well-measured genes in N. crassa protoperithecia (PP) and mycelium (Myc), relative expression levels, additions and subtractions for $95 \%$ credible intervals, and p-value, when multi-targeted primers (MTP) or oligo(dT) primers were used for reverse transcription.

Additional File 7: Comparison of expression levels of ribosomal genes in Neurospora crassa. Table of ribosomal genes and their fold change in expression in protoperithecia over mycelium, as measured by 
two-color microarray hybridization of oligo(dT)-primed cDNA and multitarget-primed CDNA

Additional File 8: Sequences of primers used for Real Time RT-PCR. Table listing the gene measured, and the forward primer and reverse primer used.

\section{Acknowledgements}

This work was supported by funding from Yale University.

\section{Author details}

${ }^{1}$ Department of Ecology and Evolutionary Biology, Yale University, 165 Prospect St, New Haven, CT 06511, USA. ${ }^{2}$ Department of Ecology and Evolutionary Biology, Program in Computational Biology and Bioinformatics, and Microbiology Graduate Program, Yale University, 165 Prospect St, New Haven, CT 06511, USA

\section{Authors' contributions}

JPT, AA, and TAC conceived and designed the experiments. JPT and FLP designed and developed the MTP algorithm. AA and TAC performed the microarray profiling. AA performed RNA tag profiling. ZW performed Real Time PCR validations. AA, JPT and FLP analyzed the data. AA and JPT wrote the manuscript. All authors read and approved the final manuscript.

Received: 25 June 2010 Accepted: 17 August 2010

Published: 17 August 2010

\section{References}

1. Bustin SA: Absolute quantification of mRNA using real-time reverse transcription polymerase chain reaction assays. J Mol Endocrinol 2000 25(2):169-193

2. Adams MD, Kelley JM, Gocayne JD, Dubnick M, Polymeropoulos MH, Xiao H, Merril CR, Wu A, Olde B, Moreno RF: Complementary DNA sequencing: expressed sequence tags and human genome project. Science 1991, 252(5013):1651-1656.

3. Velculescu VE, Zhang L, Vogelstein B, Kinzler KW: Serial analysis of gene expression. Science 1995, 270(5235):484

4. DeRisi JL, lyer VR, Brown PO: Exploring the metabolic and genetic control of gene expression on a genomic scale. Science 1997, 278(5338):680.

5. Nagalakshmi U, Wang Z, Waern K, Shou C, Raha D, Gerstein M, Snyder M: The transcriptional landscape of the yeast genome defined by RNA sequencing. Science 2008, 320(5881):1344

6. $\quad$ Yang $\mathrm{YH}$, Speed T: Design issues for cDNA microarray experiments. Nat Rev Genet 2002, 3(8):579-588

7. Wernisch L, Kendall SL, Soneji S, Wietzorrek A, Parish T, Hinds J, Butcher PD, Stoker NG: Analysis of whole-genome microarray replicates using mixed models. Bioinformatics 2003, 19(1):53-61.

8. Stahlberg A, Hakansson J, Xian XJ, Semb H, Kubista M: Properties of the reverse transcription reaction in mRNA quantification. Clinical Chemistry 2004, 50(3):509-515.

9. Graf $D$, Fisher $A G$, Merkenschlager M: Rational primer design greatly improves differential display-PCR (DD-PCR). Oxford Univ Press 1997, 25:2239.

10. Stangegaard $M$, Dufva $H$, Dufva $M$ : Reverse transcription using random pentadecamer primers increases yield and quality of resulting CDNA. BioTechniques 2006, 40(5):649-657.

11. Gonzalez JM, Robb FT: Counterselection of prokaryotic ribosomal RNA during reverse transcription using non-random hexameric oligonucleotides. J Microbiol Methods 2007, 71(3):288-291.

12. Talaat AM, Hunter P, Johnston SA: Genome-directed primers for selective labeling of bacterial transcripts for DNA microarray analysis. Nat Biotechnol 2000, 18(6):679-682.

13. Armour CD, Castle JC, Chen R, Babak T, Loerch P, Jackson S, Shah JK, Dey J, Rohl CA, Johnson JM: Digital transcriptome profiling using selective hexamer priming for CDNA synthesis. Nature Methods 2009, 6(9):647-649.

14. Matveeva OM, Shabalina SA: Intermolecular mRNA-rRNA hybridization and the distribution of potential interaction regions in murine 18S rRNA Nucl Acids Res 1993, 21:1007-1007.
15. Marzluf GA: Genetic regulation of nitrogen metabolism in the fungi. Microbiol Mol Biol Rev 1997, 61(1):17-32.

16. Bolton MD, Thomma B: The complexity of nitrogen metabolism and nitrogen-regulated gene expression in plant pathogenic fungi. Physiol Mol Plant Pathol 2008, 72(4-6):104-110.

17. Donofrio NM, Oh Y, Lundy R, Pan H, Brown DE, Jeong JS, Coughlan S, Mitchell TK, Dean RA: Global gene expression during nitrogen starvation in the rice blast fungus, Magnaporthe grisea. Fungal Genet Biol 2006 43(9):605-617.

18. Mueller T, Avolio M, Olivi M, Benjdia M, Rikirsch E, Kasaras A, Fitz M, Chalot M, Wipf D: Nitrogen transport in the ectomycorrhiza association: The Hebeloma cylindrosporum-Pinus pinaster model. Phytochemistry 2007, 68(1):41-51.

19. Lucic E, Fourrey C, Kohler A, Martin F, Chalot M, Brun-Jacob A: A gene repertoire for nitrogen transporters in Laccaria bicolor. New Phytol 2008, 180(2):343-364.

20. Manginot C, Roustan $J$, Sablayrolles JM: Nitrogen demand of different yeast strains during alcoholic fermentation. Importance of the stationary phase. Enzyme Microb Technol 1998, 23(7-8):511-517.

21. Backhus LE, DeRisi J, Brown PO, Bisson LF: Functional genomic analysis of a commercial wine strain of Saccharomyces cerevisiae under differing nitrogen conditions. FEMS Yeast Res 2001, 1(2):111-125.

22. Perkins DD, Barry EG: The cytogenetics of Neurospora. Adv Genet 1977 19:133-285.

23. Delange AM, Griffiths AJF: Meiosis in Neurospora crassa .I. the Isolation of recessive mutants defective in the production of viable ascospores. Genetics 1980, 96(2):367-378.

24. Raju NB: Genetic control of the sexual cycle in Neurospora. Mycol Res 1992, 96:241-262.

25. Gasch AP, Spellman PT, Kao CM, Carmel-Harel O, Eisen MB, Storz G, Botstein D, Brown PO: Genomic expression programs in the response of yeast cells to environmental changes. Mol Biol Cell 2000, 11(12):4241-4257.

26. Nam DK, Lee S, Zhou GL, Cao XH, Wang C, Clark T, Chen JJ, Rowley JD, Wang SM: Oligo(dT) primer generates a high frequency of truncated CDNAs through internal poly(A) priming during reverse transcription. Proc Natl Acad Sci USA 2002, 99(9):6152-6156.

27. Townsend J: Resolution of large and small differences in gene expression using models for the Bayesian analysis of gene expression levels and spotted DNA microarrays. BMC Bioinformatics 2004, 5(1):54.

28. Dieckmann CL, Tzagoloff A: Assembly of the mitochondrial membrane system. J Biol Chem 1985, 260:1513-1520.

29. Ellis TP, Helfenbein KG, Tzagoloff A, Dieckmann CL: Aep3p stabilizes the mitochondrial bicistronic mRNA encoding subunits 6 and 8 of the $\mathrm{H}$ t-translocating ATP synthase of Saccharomyces cerevisiae. J Biol Chem 2004, 279(16):15728

30. Manthey GM, McEwen JE: The product of the nuclear gene PET309 is required for translation of mature mRNA and stability or production of intron-containing RNAs derived from the mitochondrial COX1 locus of Saccharomyces cerevisiae. EMBO J 1995, 14(16):4031.

31. Butow RA, Zhu H, Perlman $\mathrm{P}$, Conrad-Webb $\mathrm{H}$ : The role of a conserved dodecamer sequence in yeast mitochondrial gene expression. Genome 1989, 31(2):757-760

32. Sambrook J, Fritsch EF, Maniatis T: Selection of poly(A)+ RNA by Oligo (dT)-cellulose chromatography. Molecular cloning Cold Spring Harbor Laboratory Press Cold Spring Harbor, NY 1989, 1:7.26-27.29.

33. Kingston R: Preparation of Poly(A)+ RNA. Current Protocols in Molecular Biology New York: John Wiley \& SonsAusubel FM, Brent R, Kingston RE, Moore DD, Seidman JG, Smith JA, Struhl K 1997, 4.5.1-4.5.3.

34. Wang SM, Fears SC, Zhang L, Chen JJ, Rowley JD: Screening poly (dA/dT)cDNAs for gene identification. Proc Natl Acad Sci USA 2000, 97(8):4162.

35. Galagan JE, Calvo SE, Borkovich KA, Selker EU, Read ND, Jaffe D, FitzHugh W, Ma LJ, Smirnov S, Purcell S, et al: The genome sequence of the filamentous fungus Neurospora crassa. Nature 2003, 422(6934):859-868.

36. Dunlap JC, Borkovich KA, Henn MR, Turner GE, Sachs MS, Glass NL, McCluskey K, Plamann M, Galagan JE, Birren BW, et al: Enabling a community to dissect an organism: overview of the Neurospora functional genomics project. Adv Genet 2007, 57:49-96.

37. Brauer MJ, Yuan J, Bennett BD, Lu WY, Kimball E, Botstein D, Rabinowitz JD: Conservation of the metabolomic response to starvation across two divergent microbes. Proc Natl Acad Sci USA 2006, 103(51):19302-19307. 
38. Lagunas R, Dominguez C, Busturia A, Saez MJ: Mechanisms of appearance of the Pasteur effect in Saccharomyces cerevisiae: inactivation of sugar transport systems. J Bacteriol 1982, 152(1):19-25.

39. Mehdi K, Penninckx MJ: An important role for glutathione and gammaglutamyltranspeptidase in the supply of growth requirements during nitrogen starvation of the yeast Saccharomyces cerevisiae. Microbiology 1997, 143:1885-1889.

40. Cooper TG, Ferguson D, Rai R, Bysani N: The GLN3 gene product is required for transcriptional activation of allantoin system gene expression in Saccharomyces cerevisiae. J Bacteriol 1990, 172(2):1014-1018.

41. Kron SJ, Styles CA, Fink GR: Symmetrical cell-division in pseudohyphae of the yeast Saccharomyces cerevisiae. Mol Biol Cell 1994, 5(9):1003-1022.

42. Smith DG, Garcia-Pedrajas MD, Gold SE, Perlin MH: Isolation and characterization from pathogenic fungi of genes encoding ammonium permeases and their roles in dimorphism. Molecular Microbiology 2003, 50(1):259-275.

43. Goodrich-Tanrikulu M, Howe K, Stafford A, Nelson MA: Changes in fatty acid composition of Neurospora crassa accompany sexual development and ascospore germination. Microbiology 1998, 144:1713-1720.

44. Bell-Pedersen D, Shinohara ML, Loros JJ, Dunlap JC: Circadian clockcontrolled genes isolated from Neurospora crassa are late night- to early morning-specific. Proc Natl Acad Sci USA 1996, 93(23):13096-13101.

45. Bobrowicz P, Pawlak R, Correa A, Bell-Pedersen D, Ebbole DJ: The Neurospora crassa pheromone precursor genes are regulated by the mating type locus and the circadian clock. Molecular Microbiology 2002, 45(3):795-804.

46. Warner JR: The economics of ribosome biosynthesis in yeast. Trends Biochem Sci 1999, 24(11):437-440.

47. Viswanath-Reddy M, Turian G: Physiological changes during protoperithecial differentiation in Neurospora tetrasperma. Physiol Plant 1975, 35(2):166-174.

48. Kraft C, Deplazes A, Sohrmann M, Peter M: Mature ribosomes are selectively degraded upon starvation by an autophagy pathway requiring the Ubp3p/Bre5p ubiquitin protease. Nat Cell Biol 2008, 10(5):602-610.

49. Malgoyre A, Banzet S, Mouret C, Bigard A, Peinnequin A: Quantification of low-expressed mRNA using 5' LNA-containing real-time PCR primers. Biochem Biophys Res Commun 2007, 354(1):246-252.

50. Issel-Tarver L, Christie KR, Dolinski K, Andrada R, Balakrishnan R, Ball CA, Binkley G, Dong S, Dwight SS, Fisk DG: Saccharomyces Genome Database. Methods Enzymol 2002, 350:329.

51. Townsend JP, Cavalieri D, Hartl DL: Population genetic variation in genome-wide gene expression. Mol Biol Evol 2003, 20(6):955-963.

52. Mewes HW, Frishman D, Guldener U, Mannhaupt G, Mayer K, Mokrejs M, Morgenstern B, Munsterkotter M, Rudd S, Weil B: MIPS: a database for genomes and protein sequences. Nucl Acids Res 2002, 30(1):31-34

53. Townsend JP, Hartl D: Bayesian analysis of gene expression levels: statistical quantification of relative mRNA level across multiple strains or treatments. Genome Biol 2002, 3(12), research0071.0071-0071.0016.

54. Kanehisa M, Goto S: KEGG: Kyoto Encyclopedia of Genes and Genomes. Nucl Acids Res 2000, 28(1):27-30.

55. Zhang Z, Townsend JP: The filamentous fungal gene expression database (FFGED). Fungal Genet Biol 2009, 47(3):199-204.

56. Barrett T, Edgar R: Gene expression omnibus: microarray data storage, submission, retrieval, and analysis. Methods Enzymol 2006, 411:352-369.

57. Clark AT, Guilmette JM, Renstrom D, Townsend JP: RNA extraction, probe preparation, and competitive hybridization for transcriptional profiling using Neurospora crassa long-oligomer DNA microarrays. Fungal Genet Rep 2008, 55:18-28.

58. Kasuga T, Townsend JP, Tian C, Gilbert LB, Mannhaupt G, Taylor JW, Glass NL: Long-oligomer microarray profiling in Neurospora crassa reveals the transcriptional program underlying biochemical and physiological events of conidial germination. Nucl Acids Res 2005, 33(20):6469-6485.

doi:10.1186/1471-2164-11-477

Cite this article as: Adomas et al: Multi-targeted priming for genomewide gene expression assays. BMC Genomics 2010 11:477.

\section{Submit your next manuscript to BioMed Central and take full advantage of:}

- Convenient online submission

- Thorough peer review

- No space constraints or color figure charges

- Immediate publication on acceptance

- Inclusion in PubMed, CAS, Scopus and Google Scholar

- Research which is freely available for redistribution 